
cultures

Les cahiers de l'Acedle

9-1 | 2012

Varia : plurilinguisme et transdisciplinarité

\title{
L'auto-évaluation comme facteur d'évolution du rapport à l'apprentissage des langues
}

\author{
Nadine Normand-Marconnet
}

\section{(2) OpenEdition}

Journals

Édition électronique

URL : http://journals.openedition.org/rdlc/2476

DOI : $10.4000 /$ rdlc. 2476

ISSN : 1958-5772

Éditeur

ACEDLE

Édition imprimée

Date de publication : 1 avril 2012

\section{Référence électronique}

Nadine Normand-Marconnet, «L'auto-évaluation comme facteur d'évolution du rapport à

l'apprentissage des langues », Recherches en didactique des langues et des cultures [En ligne], 9-1 | 2012, mis en ligne le 01 avril 2012, consulté le 16 mars 2020. URL : http://journals.openedition.org/rdlc/ 2476 ; DOI : https://doi.org/10.4000/rdlc.2476

\section{(c) $\Theta \Theta \Theta$}

Recherches en didactique des langues et des cultures is licensed under a Creative Commons AttributionNonCommercial-NoDerivatives 4.0 International License 


\title{
L'auto-évaluation comme facteur d'évolution du rapport à l'apprentissage des langues
}

\author{
Nadine Normand-Marconnet \\ Monash University, Australie
}

\section{Résumé}

Les politiques éducatives de nombre d'Etats en et hors d'Europe sont aujourd'hui axées sur la mise en place d'une éducation plurilingue. En Europe, mais aussi sur les continents américain et asiatique, de nombreux Etats affichent la volonté de mettre en place une éducation plurilingue. Elle a pour but de permettre à chacun de prendre conscience de ses compétences plurilingues et pluriculturelles et de les faire évoluer tout au long de la vie. Dans ce cadre, nous retiendrons la question de l'auto-évaluation comme facteur d'évolution du rapport à l'apprentissage des langues. En quoi les dérivés du Cadre européen commun de référence (Cecr) que sont les portfolios pour l'apprenant (Portfolio européen des Langues, ou PEL) et pour l'enseignant en formation (Portfolio européen pour les enseignants en langues en formation initiale, ou Pepelf) peuvent-ils placer l'auto-évaluation au cour d'une didactique du plurilinguisme? Quels sont les obstacles qu'une telle démarche peut susciter chez les acteurs concernés? En nous référant à une étude de terrain menée auprès d'enseignants de français en Iran, nous tenterons d'apporter des réponses à ces questions et de montrer qu'un travail sur les représentations sociales est indispensable pour faire évoluer les opinions et les pratiques en matière d'auto-évaluation.

\section{Mots-Clés}

auto-évaluation, formation des enseignants, portfolios, représentations sociales, enseignement-apprentissage du français, Iran 


\begin{abstract}
In many states both within and outside of Europe, education authorities are trying to implement plurilingual educational systems. The main target is to enable everyone to be aware of their plurilingual and pluricultural competences and to improve them throughout their lifetime. In this paper, we will analyse how self-assessment is supposed to help change people's opinion about language learning. How can the European Portfolio of Languages (EPL) and the European Portfolio for Student Teachers of Languages (Epostl), two documents associated with the Common European Framework of Reference for Languages (Cefr), contribute to put self-assessment at the heart of plurilingual education? What are the difficulties encountered by the different actors involved? This study, carried out with Iranian teachers, tries to provide some answers and show that working on socially construed beliefs linked to the self-assessment process is a first step in bringing change to opinions and practices.
\end{abstract}

\title{
Keywords
}

self-assessment, teacher training, portfolios, social representations, teaching-learning French, Iran

\section{Introduction}

Ces quarante dernières années, des instances comme le Conseil de l'Europe et la Commission Européenne sont à l'origine de profonds changements en matière de politiques éducatives et de conception de l'enseignement-apprentissage des langues. Le Conseil Européen qui s'est tenu à Lisbonne en 2000 a entériné l'entrée de l'Europe dans "l'ère du savoir" et a fait de l'apprentissage tout au long de la vie un principe directeur qui va bien audelà des simples politiques éducatives. Le mémorandum adopté reprend et développe le postulat du livre blanc européen selon lequel la société du futur sera une "société cognitive" dans laquelle l'éducation et la formation deviendront davantage "les principaux vecteurs d'identification, d'appartenance, de promotion sociale et d'épanouissement personnel" (Commission Européenne, 1995 : 2). 
La promotion d'une éducation plurilingue constitue donc le défi majeur de la plupart des États européens aujourd'hui. Dans le contexte actuel d'une mobilité académique et professionnelle croissante, de nombreux Etats européens affichent la volonté de promouvoir une éducation plurilingue. Le concept de plurilinguisme est proche et pourtant essentiellement différent de celui de multilinguisme. Ce dernier renvoie en effet à la présence d'une "variété de langues" dans une zone géographique précise dans laquelle un individu peut rester monolingue toute sa vie, malgré la multiplicité de langues qui l'entoure. De même, il ne faut pas le confondre avec un polyglotte, "locuteur plurilingue particulièrement expert" (Beacco, 2005 : 19).

Les recherches récentes menées dans ce domaine montrent que favoriser le plurilinguisme revient à permettre l'enrichissement du "répertoire plurilingue" de chacun, répertoire composé des langues ou variétés de langue qu'il a acquises selon des modalités particulières (apprentissage scolaire, familial, professionnel...) maîtrisées à différents niveaux et pour des compétences particulières (lecture, conversation, etc.). L'éducation plurilingue vise donc à provoquer une prise de conscience par l'apprenant du répertoire qu'il possède et de sa capacité à le faire évoluer en fonction des circonstances. Ainsi se développe "une compétence plurinlingue et pluriculturelle qui ne résulte pas de la simple addition de compétences monolingues" mais plutôt de combinaisons et d'alternances entre celles-ci (Coste, Moore \& Zarate, 1997 : 13).

Dans ce cadre, nous examinerons en quoi des pratiques évaluatives innovantes comme l'auto-évaluation peuvent contribuer à la construction d'une didactique du plurilinguisme. Le Portfolio Européen des Langues (PEL) et le Portfolio Européen pour les Enseignants en Langues en Formation initiale (Pepelf) serviront d'exemples d'instruments favorisant l'autonomisation de l'apprentissage et la valorisation de trajectoires plurilingues, en dépit de difficultés prévisibles lors de leur mise en place. Celles-ci sont souvent liées au poids de la culture éducative et des représentations sociales qu'elle véhicule, comme le montre l'expérimentation que nous avons menée en Iran. 


\section{L'auto-évaluation au cœur d'une didactique du plurilinguisme}

\subsection{Cadre théorique}

Le Cadre Européen Commun de Référence (Cecr) et les récents travaux du Conseil de l'Europe ont permis des évolutions notables en matière de didactique des langues. Au centre des recherches qui ont conduit à l'élaboration des textes de référence comme le Cecr se trouve le concept majeur d"'apprendre à apprendre". Il semble aujourd'hui évident que pour renforcer la capacité de chaque individu à apprendre tout au long de sa vie, il faut lui permettre de développer sa capacité à être autonome dans son apprentissage. Paradoxalement, la notion combinée d'"apprentissage autonome" au cœur de la réflexion européenne n'est pas définie précisément dans le Cecr (qui s'attarde en revanche longuement sur celles de compétence et de tâche communicative), ni même dans le PEL. Or c'est bien du côté de l'évaluation, et plus particulièrement de l'auto-évaluation qu'il faut chercher le fil conducteur de ce processus impliquant directement l'apprenant dans la détermination de ses besoins, de ses objectifs, des moyens à mettre en œuvre, autrement dit "la capacité à prendre en charge son propre apprentissage" (Holec, 1981 : 3).

De fait, depuis les années 70, les recherches se sont multipliées sur l'auto-évaluation qui figure en bonne place dans la littérature scientifique consacrée à l'évaluation formative. Introduit par Scriven (1967), ce concept a été progressivement élargi pour englober trois types de régulation (Allal, 1988) : la régulation interactive, la régulation rétroactive et la régulation proactive. Les recherches actuellement menées dans la sphère francophone montrent que l'évaluation formative dans sa forme "interactive" (qui englobe l'autoévaluation notamment par la mise en place d'un portfolio), est reconnue comme l'un des moyens les plus efficaces en matière d'activation de ces processus de régulation métacognitive (Allal, 2011). De nombreux travaux ont permis de définir précisément les bénéfices apportés par l'auto-évaluation : elle renforce l'autonomie et la motivation des apprenants (Holec, 1991) en favorisant un apprentissage actif (Harris, 1997). Elle leur permet également de prendre conscience de leurs stratégies d'apprentissage (Duda \& Riley, 1990 ; Wenden, 2002), de valoriser leurs parcours d'apprentissage et leurs expériences interculturelles et de mettre en évidence ceux qui ne font pas l'objet d'une certification (Coste, Moore \& Zarate, 1997). 
De façon synthétique, l'auto-évaluation peut être définie comme relevant d'une

réflexion métacognitive qui met en interaction le cadre d'organisation de l'activité et le réseau de représentations à travers lequel le sujet interprète la réalité. Elle entremêle les opérations d'évaluation et de mouvements de prise de distance du sujet avec des objets qu'il a produit et qu'il observe comme traces de son action (Campanale, 1997 : 6).

Quels que soient les outils mis en œuvre, une démarche auto-évaluative transforme indéniablement la relation enseignant-enseigné : le premier est appelé à guider ce processus métacognitif en invitant le second à développer et mettre en œuvre des conduites réflexives et autonomes. Ce changement de paradigme qui tend à faire évoluer profondément le rapport à l'apprentissage des acteurs peut se réaliser dès lors que les obstacles, à la fois techniques et psychologiques, ont été surmontés. Selon l'inventaire dressé par Holec (1981), l'apprenant doit tout d'abord être capable d'apprécier sa performance en termes de "réussi/non réussi", c'est-à-dire, d'observer celle-ci et de l'analyser puis la comparer à une performance témoin. Il doit pouvoir interpréter ses résultats, autrement dit déterminer les performances à prendre en compte, comparer les résultats atteints aux résultats prévus, porter et adapter son jugement. Pour atteindre ces objectifs, il doit apprendre à objectiver ses performances, définir des catégories d'analyse pertinentes, mobiliser des connaissances descriptives, considérer les conditions de réalisation de ses performances, définir les objectifs avec précision ainsi que ses seuils de satisfaction. Un cadre de formation est donc nécessaire pour permettre à l'apprenant d'acquérir et de mettre en pratique des connaissances en matière de différences entre évaluation externe et interne, et de mieux apprécier le rôle positif de l'erreur et l'importance relative du risque de mauvaise appréciation. En second lieu, et sur le plan psychologique, il faudra le former à faire évoluer ses représentations sur l'apprentissage et le rôle qu'il a à jouer ainsi que l'amener à réfléchir aux contraintes sociologiques qui pèsent sur lui. En d'autres termes, un "déconditionnement" est nécessaire pour que l'apprenant cesse de considérer l'évaluation comme une sanction externe et soit peu à peu amené à la penser comme un outil de contrôle sur son action d'apprentissage, et partant, en fasse un vecteur privilégié d'autonomisation. Harris (1997) complète cette analyse en insistant sur les facteurs socio-culturels qui influencent la perception du progrès chez les étudiants. D'après lui, dans de nombreux pays, la méthodologie d'enseignement d'une langue étrangère est calquée sur 
celle des autres matières et l'évaluation se fait sur des bases sommatives. Cela incite les apprenants à évaluer leur progression en langue à l'aune de leur maîtrise lexicale et grammaticale et non en termes de performance de la communication. En d'autres termes, quand ils se trouvent confrontés à une démarche d'auto-évaluation, il leur est difficile de passer d'une analyse "quantitative et déclarative" (fondée sur les notions de savoirs [knowledge] et de faits observables [facts]) à une analyse "procédurale et qualitative" (fondée sur les notions de performance [performance] et de savoir-faire [skills], p.15).

\subsection{Le PEL et le Pepelf, exemples de portfolios d'auto-évaluation à destination des apprenants et des enseignants}

Le PEL (portfolio pour les apprenants) comme le Pepelf (pour les enseignants en formation) sont des outils dérivés du Cecr, conçus pour être adaptés aux besoins spécifiques des autorités éducatives qui les adoptent et déclinés en fonction des contextes locaux. Dans le prolongement de l'approche actionnelle privilégiée par les instances européennes, ils ont également en commun d'être centrés sur l'auto-évaluation et construits en partie au moyen de descripteurs de compétences de type dit "can do" (je suis capable de). Quelle qu'en soit l'utilisation faite, ils restent la propriété de leur utilisateur.

Le PEL a été lancé en 2001 à la suite de la publication du Cecr; quelles que soient les adaptations effectuées et validées par la Division des Politiques Linguistiques du Conseil de l'Europe (Division des politiques linguistiques, 2000), les portfolios en vigueur ont pour but d'encourager le développement de l'autonomie des apprenants. Pour ce faire, le PEL propose dans la partie "passeport des langues" une grille d'auto-évaluation basée sur les 6 niveaux de compétence définis dans le Cecr. Il permet de définir son profil linguistique en lien avec la grille pour l'auto-évaluation du Cecr, de lister dans une grille prédéfinie les expériences d'apprentissage linguistiques et interculturels significatives, puis de récapituler les diplômes et certificats obtenus. C'est cette première partie qui est généralement adaptée selon le niveau et le lieu d'utilisation: selon les pays et les niveaux d'apprentissage (primaire, secondaire, lycée, formation pour adultes, etc.), les concepteurs vont utiliser des termes différents pour les différentes compétences (écouter, lire, prendre part à une conversation, s'exprimer oralement en continu et écrire), ainsi qu'une échelle de notation particulière (1 à 6 par ordre croissant du plus difficile au plus facile, ou icônes tels que $: ;$, $\odot$,, , etc.). La 
"biographie langagière" est conçue pour favoriser l'implication de l'apprenant dans la planification de son apprentissage au moyen notamment de listes de repérage qui proposent un inventaire des tâches d'apprentissage correspondant aux exigences des curricula officiels. Le "dossier" contient les documents retenus pour illustrer les expériences et les acquis mentionnés dans les autres parties. Ces trois composantes permettent au PEL de remplir la double fonction qui lui a été assignée à l'origine. En premier lieu, une fonction pédagogique car le PEL doit mettre en valeur les démarches d'apprentissage, l'évaluation formative, les motivations et les occasions d'apprentissage de l'apprenant pour valoriser son expérience plurilingue et pluriculturelle. En second lieu, une fonction de documentation et de présentation qui concerne les résultats et les produits de l'apprentissage que des acteurs extérieurs (parents, employeurs, etc.) peuvent consulter aisément.

Àl'heure actuelle, 118 versions ont été validées dans 34 Etats membres du Conseil de l'Europe ce qui montre la large diffusion de cet outil favorisant le plurilinguisme dans la formation pour adultes (Little, 2009) comme dans le système scolaire. En France, comme le soulignent Castellotti et Moore, les activités proposées dans le PEL Collège

donnent corps au plurilinguisme et le démystifient, en le rapprochant de l'expérience des élèves et en l'insérant dans leur quotidien scolaire et social. Les élèves peuvent alors se vivre comme les acteurs de ce plurilinguisme, ou du moins confirmer qu'ils sont en passe de le devenir (Castellotti \& Moore, 2006 : 66).

C'est en partie pour répondre aux questions des enseignants chargés de mettre en pratique le PEL dans leurs classes qu'a été conçu le Pepelf (Newby, 2007). Pour l'heure seulement disponible en version anglaise et française, il a été développé par le Centre Européen pour les Langues Vivantes à partir des documents de référence que sont le Cecr, le PEL et le Profil européen pour la formation des enseignants de langue. Comme ces derniers, le Pepelf (Portfolio européen pour les enseignants en langue en formation initiale) constitue un instrument de promotion de l'éducation à la citoyenneté démocratique, de la valorisation des compétences plurilingues et pluriculturelles et de l'autonomie de l'apprentissage. Il reprend les éléments centraux du Profil, étude précédemment réalisée par une équipe de l'université de Southampton qui a permis de définir un cadre de référence pour l'adoption de programmes de formation d'enseignants de langue harmonisés à l'échelle européenne. Au moyen de 193 descripteurs classés en 7 catégories principales (contexte, méthodologie, 
ressources, préparation du cours, faire cours, apprentissage autonome, évaluation de l'apprentissage), le Pepelf propose un modèle de catégorisation pour la spécification des compétences didactiques que le futur enseignant de langues doit s'efforcer d'acquérir tout au long de sa formation initiale. À l'image du PEL, il comporte une section de "déclaration personnelle", une section d'auto-évaluation composée de descripteurs de compétences et un dossier personnel, complétés par un glossaire, un index et un guide d'utilisation.

Ses objectifs sont clairement en relation avec ceux poursuivis par le PEL pour les apprenants. En effet, le Pepelf se propose d'encourager les futurs enseignants de langue en formation initiale. Il vise à les faire réfléchir sur leurs compétences, à les aider à se préparer aux diverses situations d'enseignement qu'ils peuvent connaître, à faciliter les échanges entre eux et avec leurs formateurs. De plus, en favorisant une démarche d'auto-évaluation de leurs compétences en cours d'acquisition, il doit permettre aux futurs enseignants de langue de prendre conscience de leurs forces et de leurs faiblesses en matière d'enseignement. Enfin, il se présente comme un instrument permettant de visualiser les progrès réalisés. Toutefois, les concepteurs insistent sur le fait que le Pepelf ne peut faire office de simple référentiel de compétence ou de liste de contrôle utilisable en fin de période de formation. Comme le Cecr et le PEL, il est appelé à être adapté à chaque contexte particulier et il appartient à son utilisateur. Projet pilote en cours d'expérimentation dans plusieurs Etats européens entre 2008 et 2011, il devrait donner lieu à la publication d'un rapport qualitatif sur le pilotage contenant des exemples de "bonnes pratiques" liées à ce portfolio et sur les possibilités d'application ultérieure.

\section{Auto-évaluation et culture éducative : quels obstacles ? Quelles pistes de solution?}

\subsection{Des difficultés prévisibles pour les enseignants : une étude de cas en Iran}

Quel que soit le contexte éducatif, il semble que les difficultés et les résistances rencontrées chez les enseignants dès lors qu'ils sont confrontés à une démarche auto-évaluative soient assez semblables. Nous en voulons pour preuve les résultats obtenus au terme d'une enquête de deux ans menée auprès d'enseignants iraniens, que nous avons comparés à des données obtenues en Europe. Il est ainsi frappant de constater que les enseignants porteurs d'une 
culture éducative dite "traditionnelle" comme celle qui prévaut en Iran (i.e. caractérisée entre autres par une transmission magistrale des savoirs et une relation pédagogique fortement hiérarchisée) ont finalement les mêmes questionnements et les mêmes réactions que ceux de leurs collègues occidentaux face à l'auto-évaluation. Ce constat nous conforte dans la nécessité de prendre en compte les représentations sociales des acteurs impliqués et de les accompagner dans ce type de démarche induisant des pratiques pédagogiques innovantes.

C'est dans ce sens que nous avons mené notre recherche en Iran dans le cadre d'une thèse de doctorat (Normand-Marconnet 2008). En tant que conseillère pédagogique, nous avons suivi un groupe de 97 enseignants qui ont créé et expérimenté, sur 18 mois, un ensemble de fiches auto-évaluatives à destination de leurs apprenants. Ces fiches étaient composées de descripteurs de compétences sur le modèle de ceux utilisés dans les listes de repérage du PEL. Notre démarche à visée pragmatique a consisté à combiner expérimentation, suivi des réactions des enseignants (par entretiens et questionnaires) et mise en place d'ateliers de formation réflexive pour permettre une adaptation de la démarche au contexte local. Cet article se concentre uniquement sur la partie concernant l'analyse des résultats relatifs aux représentations des enseignants.

À l'issue de l'expérimentation, un bilan a pu être dressé sur la base de questionnaires remis aux enseignants concernés, dans l'objectif de mettre à jour l'évolution de leurs opinions sur l'auto-évaluation. Pour ce faire, certaines propositions qui figuraient dans un questionnaire initial conduit en début de l'expérimentation ont été conservées, ainsi que des avis qui semblaient significatifs car ayant été formulés à plusieurs reprises lors de réunions collégiales ou d'entretiens en face à face avec des enseignants. Ont été ajoutées des questions destinées à provoquer une prise de conscience sur des points qui paraissaient avoir été occultés pendant l'expérimentation. Sur un total de 25 propositions $^{1}, 7$ étaient des questions de type fermé (oui/non/je ne sais pas) et 18 de type échelle selon le modèle de Lickert qui permettent de mieux cerner les nuances d'opinion. En effet, pour chaque item, les personnes interrogées sont invitées à se positionner en choisissant l'un des échelons suivants : "tout à fait d'accord", "plutôt d'accord", "sans opinion", "plutôt en désaccord",

\footnotetext{
${ }^{1}$ Cf. annexe.
} 
"tout à fait en désaccord". Ils sont respectivement codés de 1 à 5 pour donner lieu à une exploitation des données par addition des scores pour chaque item-opinion multipliée par le coefficient correspondant, divisée ensuite par le nombre de réponses pour obtenir la moyenne de l'item. Cette moyenne sera le reflet de l'opinion globale de l'échantillon testé : plus elle se rapproche de 1 et plus elle indique un fort taux d'adhésion parmi les sondés.

Sur les 97 questionnaires envoyés aux enseignants impliqués dans l'expérimentation, 46 seulement ont été retournés malgré plusieurs relances. Les données quantitatives obtenues montrent que parmi les représentations qui ont évolué, celle qui associait l'importance de la démarche à la nouveauté pédagogique paraît moindre qu'au début (moyenne de 1,84 ). Nous y voyons le signe probable qu'après plusieurs mois de pratique, la fiche d'auto-évaluation a perdu son caractère de "gadget moderne". Mais c'est surtout le changement de discours sur une pseudo incapacité des apprenants à s'auto-évaluer qui est particulièrement notable : en premier lieu, les enseignants considèrent que cette inaptitude n'est pas le fait de la condition intrinsèque de l'apprenant. Pour les propositions stipulant qu'ils "ne sont pas objectifs sur leur niveau" ou qu'ils "ne comprennent pas à quoi ça sert", les moyennes relevées sont respectivement de 2,81 et 2,97 , ce qui illustre une forte divergence de la part des sondés face à cette affirmation. Selon les enseignants interrogés, les difficultés des apprenants en la matière résulterait de causes externes, liées au contexte local et notamment parce qu'ils "donnent plus d'importance aux examens et aux notes" (moyenne de 1,92) et qu'"ils ne sont pas habitués à réfléchir à ces questions et à donner leur avis".

De plus, les enseignants n'adhèrent pas massivement à la proposition stipulant qu'ils seraient les seuls experts en la matière (moyenne de 3,15 à l'item "seul l'enseignant est expérimenté pour juger le niveau des apprenants"). Par rapport aux opinions émises dans les premiers questionnaires, ce résultat marque un changement remarquable en matière de délégation du pouvoir à évaluer. De façon concrète, il semblerait que les enseignants de notre échantillon aient pu vérifier par eux-mêmes ce que d'autres études menées en Europe, notamment au Crapel de Nancy sous la direction de Holec ont montré clairement : les apprenants de niveau plus élevé ont tendance à se sous-estimer, alors que ceux de niveau plus faible ont plutôt tendance à se surestimer. D'après lui, "le niveau des connaissances n'améliore pas la qualité intrinsèque de l'auto-évaluation, mais modifie le degré d'exigence sur la base duquel 
l'apprenant sélectionne ses critères et ses seuils d'évaluation" (Holec, 1981 : 45). En outre, le fait de s'être réellement impliqués conduit les enseignants à considérer que la réussite de la démarche en Iran est plus liée à leur propre implication qu'à l' intervention des autorités supérieures : ils semblent plus adhérer à la proposition selon laquelle "il faudrait que les enseignants qui commencent à se familiariser avec l'auto-évaluation introduisent peu à peu cette démarches dans les instituts, écoles et universités où ils travaillent sans attendre l'appui du ministère" (moyenne de 1,52) qu'à celle qui stipule qu'"il faudrait qu'elle soit imposée dans les programmes scolaires par le ministère de l'Education" (moyenne de 1,98). Par ailleurs, ils disent préférer un travail de collaboration entre pairs plutôt que de la formation associant des experts extérieurs (moyenne de 1,90).

Àces traces d'évolution s'ajoutent des représentations qui se confirment. Elles concernent la volonté des enseignants de continuer la démarche (64\% de "non" à l'item "je n'ai pas envie de continuer", avec ou sans le soutien de leur direction (52\% de "oui" pour "j'ai envie de continuer cette démarche de façon autonome"). Ils se disent également persuadés des bénéfices tirés de l'auto-évaluation par l'ensemble de l'institution et estiment qu'il est nécessaire de continuer cette démarche pour améliorer l'ensemble des programmes" (moyenne de 1,41). En outre, ils partagent le sentiment qu'elle amène l'enseignant à prendre du recul pour améliorer ses cours (moyenne de 1,42). Au vu de ces éléments, il serait légitime de conclure à une transformation des représentations sur le rôle du professeur dans la démarche d'auto-évaluation, et par conséquent à une modification de la relation pédagogique. Or, il faut noter le sentiment tenace chez les enseignants qu'une telle démarche n'a pas pour objectif principal d'instaurer une relation différente avec les apprenants. De fait, ils déclarent ne pas avoir constaté de modification dans le mode de fonctionnement avec leurs élèves, pas plus qu'un changement d'attitude de ceux-ci à leur égard : 50\% à peine répondent "oui" à la proposition "j'ai l'impression d'avoir une relation différente avec mes apprenants", et 49\% ont l'impression que les "apprenants [les] considèrent autrement". Ils reconnaissent toutefois que la démarche d'auto-évaluation a une incidence sur la réflexion des apprenants quant à leur apprentissage: "il est nécessaire de continuer pour faire réfléchir les apprenants à leur apprentissage" obtient la moyenne la plus positive $(1,31)$. Cependant, ils ne se déclarent pas totalement convaincus par l'apparition d'une motivation accrue (moyenne de 1,76) ou une nécessaire association des apprenants à la démarche en 
général (moyenne de 1,79). Il semblerait donc qu'à l'issue de 18 mois de mise en pratique d'une démarche auto-évaluative, les enseignants de notre échantillon ne parviennent pas à entrevoir de façon claire ce que l'auto-évaluation peut apporter à l'apprenant sur le plan métacognitif, très probablement par manque de connaissance théorique sur le sujet.

\subsection{Un bilan ponctuel mais éclairant}

L'ensemble des données recueillies durant notre projet de recherche montre qu'après quelques mois d'expérimentation, la plupart des enseignants confirment leur intérêt pour l'auto-évaluation en tant que vecteur de modernité et de nouveauté dans leur enseignement. Elle est bien acceptée car elle leur permet, ainsi qu'aux apprenants, de mieux suivre la progression pédagogique de la classe et contribue ainsi à l'amélioration des programmes. Certains soulignent qu'elle les aide à mieux cibler les niveaux de compétence et favorise une plus grande implication des apprenants dans leur apprentissage. En revanche, la dissymétrie de position entre enseignants et apprenants dans le cadre de pratiques liées à l'autoévaluation semble perdurer. Les dires convergents des apprenants et des enseignants témoignent de l'inexistence en Iran d'activités de ce type; l'auto-évaluation n'est pas habituelle dans le domaine éducatif, ce qui engendre méconnaissance, risque de mauvaise interprétation de la démarche, incompétence, voire même sentiment d'anormalité. En outre, la plupart des enseignants interrogés insistent sur la nécessité de conserver un rôle prédominant dans un type de pratique normalement autocentrée sur les apprenants: les premiers revendiquent une légitimité reposant sur leur formation et sur leur expérience professionnelle pour tout ce qui touche à l'évaluation, qui leur confère un pouvoir que certains ne souhaitent pas voir remis en cause. En contrepartie, et par un effet de balancier, la capacité des apprenants à s'auto-évaluer est donc sujette à caution : elle se traduit par une propension à se surestimer pour certains et à se sous-estimer pour d'autres.

Différents indicateurs inclus dans la notion de culture éducative peuvent être mobilisés pour appuyer ce constat : les doutes émis par certains apprenants quant à la capacité intrinsèque à s'auto-évaluer et la tendance soi-disant commune à se surévaluer tiendraient paradoxalement au climat général de compétition scolaire en vigueur et au postulat d'une autorité de l'enseignant et de l'institution non contestable. Il est en effet généralement admis que seuls peuvent réussir dans le système scolaire iranien les élèves qui se conforment strictement aux 
instructions et consignes données par les représentants de l'institution scolaire. Enfin, le fait que ne soit quasiment pas exprimée l'idée qu'une démarche d'auto-évaluation favorise le dialogue entre les apprenants et les enseignants nous semble un indicateur sérieux d'une relation pédagogique plus verticale qu'horizontale qui peut constituer une source d'obstacles potentiels.

Toutefois, ces particularités propres au contexte iranien ne sont pas sans se rapprocher de certaines constatations émises en Europe. Comme le souligne Holec dès 1981, "le premier démon à exorciser est l'incapacité des apprenants à apprécier leurs connaissances" (Holec, 1981: 45). Vingt ans plus tard, le suivi du projet-pilote du PEL en Europe entre 1998 et 2000 vient conforter ce constat, comme l'illustre le rapport final établi par Schärer (2000), constitué de questionnaires retournés par les enseignants et les apprenants des 15 pays qui ont utilisé la première version du PEL à titre exploratoire. La comparaison des réponses fournies par les enseignants et les apprenants en 1999 et en 2000 (respectivement 200 et 2080, puis 370 et 5370) montrent une réelle différence de jugement entre les premiers qui se déclarent à $65 \%$ d'accord avec l'auto-évaluation de leurs élèves alors que ceux-ci ne sont que $53 \%$ à estimer que leurs professeurs ont le même avis qu'eux.

\section{Discussion : représentations sociales, réflexivité et contextualisation}

Dans un contexte particulier et sur une échelle réduite, l'étude menée en Iran illustre le poids des représentations sociales dans l'analyse des résistances qui peuvent se manifester lors de la mise en place d'une démarche d'auto-évaluation. Ces phénomènes métacognitifs complexes ont commencé à faire l'objet de recherches poussées en sciences sociales dès les années soixante. En 1961, dans son ouvrage La psychanalyse, son image et son public, le psychosociologue S. Moscovici met à jour les principes de formation et de fonctionnement des représentations sociales et avance l'explication d'un double processus. Selon lui, "l'objectivation" permet à l'individu de mettre en image et de structurer une notion abstraite ; "l'ancrage" lui permet d'incorporer des éléments moins familiers dans des catégories fonctionnelles qui lui sont connues. C'est pendant la phase d'objectivation que se crée le "noyau figuratif", organisation des éléments sous forme d'un schéma imagé qui est repris et développé par Abric (1994) et Flament (1999), au cœur d'un courant de recherche 
actuellement très actif. De "figuratif", le noyau devient "central", par opposition à des éléments dits "périphériques". Constitué d'un nombre très limité d'éléments qui lui donne sa signification (fonction génératrice) et détermine les relations entre ses éléments constitutifs (fonction organisatrice), ce noyau est doté d'une "centralité qualitative et structurale qui fait de lui l'élément unificateur et stabilisateur de la représentation" (Flament, 1999 : 227). Pour Abric (1994, : 78), "c'est par lui que se réalise et se définit l'homogénéité d'un groupe. Il est extrêmement normatif et relativement indépendant du contexte social et matériel immédiat dans lequel la représentation est mise en évidence". Parallèlement, les "éléments périphériques" ont pour caractéristiques d'être souples et flexibles, et sont donc à l'origine de modulations potentielles et de variations individualisées. En permettant le décryptage immédiat de la situation, ils rendent possible l'adaptation tout en protégeant le noyau central, par nature très résistant au changement: partie opérationnelle de la représentation, ils fonctionnent comme des schèmes prescripteurs des comportements. De façon plus large nous retiendrons que, d'après Castellotti et Moore (2002: 21) "les représentations sont constitutives de la construction identitaire, du rapport entre soi et les autres et de la construction des connaissances".

Intimement lié aux représentations sociales, le concept d'habitus nous paraît également incontournable dans l'analyse de potentielles réticences face à l'auto-évaluation. Aujourd'hui souvent assimilé à la notion de "culture éducative", l'habitus a été le pivot de l'œuvre du sociologue P. Bourdieu : empruntant ce terme à Thomas d'Aquin, il l'élargit et le définit comme un

système de dispositions durables et transposables qui, intégrant toutes les expériences passées, fonctionne à chaque moment comme une matrice de perceptions, d'appréciations et d'actions, et rend possible l'accomplissement de tâches infiniment différenciées, grâce au transferts analogiques de schèmes permettant de résoudre les problèmes de même forme (Bourdieu, 1972 : 178-179).

L'habitus se concrétiserait donc par l'intériorisation des contraintes externes et du poids de la structure sociale et expliciterait ainsi les phénomènes de reproduction. La théorie de la pratique développée par Bourdieu et ses successeurs subordonne donc la transformation de l'habitus à une transformation des structures. En didactique, des auteurs comme Perrenoud mobilisent ce concept en en faisant une "grammaire génératrice" de nos pensées et de nos 
actes, c'est-à-dire un "ensemble de dispositions intériorisées dont on ne saisit les manifestations qu'au travers des actes et des façons d'être au monde" (Perrenoud, 2001 : 137). Dans ce cadre, l'auto-évaluation, en tant que pratique favorisant l'autonomisation des apprenants apparaît bel et bien comme un "changement subversif [par la] remise en cause de la transmission des échanges, des biens symboliques, de la fonction même des enseignants, et de la représentation de l'apprenant [qu'elle opère]." (Barbot, 1990 : 94).

Aujourd'hui, la prise en compte de l'habitus scolaire et des représentations s'avère indispensable dès lors qu'il s'agit de faire évoluer les pratiques professionnelles des enseignants (Grandcolas \& Vasseur, 1999 ; Cambra Giné, 2003). En outre, la nécessité d'une formation paraît incontournable si l'on souhaite mettre en place une démarche d'autoévaluation basée sur le principe d'acteurs autonomes tels que décrit par le Conseil de l'Europe : l'enseignant doit amener l'apprenant à accepter le fait d'avoir une responsabilité équivalente à la sienne dans la co-production de savoir, pas seulement sur le plan affectif, mais également sur le plan de la prise d'initiative en matière d'organisation. En somme, l'objectif général à atteindre dès la formation initiale si possible est la mise en place d'une réflexion sur la spécificité de l'acquisition du savoir enseigné, c'est-à-dire inciter les enseignants à adopter un angle d'analyse focalisé sur l'acte d'apprendre plus que sur celui d'enseigner, et s'interroger sur ce qui facilite ou entrave l'acquisition. Nunan (1999) suggère qu'une formation sur "l'apprendre à l'apprendre" traite des points suivants : le processus d'apprentissage en général; la sensibilisation à l'importance du contexte ; la connaissance des macro-compétences et du système linguistique afin de coupler réflexion sur l'apprentissage et entraînement aux stratégies. En effet, il paraît évident que l'on ne peut valablement demander aux enseignants d'aider leurs apprenants à "se déconditionner", selon les termes de Holec, pour avoir une autre conception de l'évaluation, si eux-mêmes ne sont pas préparés à l'incontournable décentration qu'exige toute démarche auto-évaluative. Pour ce faire, de nombreuses modalités sont envisageables à partir d'une conception réflexive de la formation. Issue d'un courant de réflexion fécond dans le domaine pédagogique, celle-ci se caractérise par la volonté de concilier théorie et pratique, la centration sur celui qui est au cœur de l'action d'apprentissage, l'analyse et la prise en compte de la situation dans laquelle se déroule l'action, la confrontation de son expérience avec des pairs (Blanchard-Laville \& Fablet, 2001). 
À cette approche réflexive adoptée lors des séances de travail avec les enseignants iraniens, il nous a semblé nécessaire d'adjoindre un processus de contextualisation des outils d'autoévaluation afin que les acteurs impliqués dans la démarche se l'approprient véritablement. Ce travail d'adaptation peut revêtir plusieurs aspects, et s'inscrire dans le cadre de la formation initiale ou continue des enseignants de langues. Dans le cas de notre action menée en Iran et suite à une précédente expérience réalisée en France, nous avons posé comme principe de départ qu'il ne serait pas pertinent d'utiliser des fiches d'auto-évaluation existantes (dans les manuels ou dans les portfolios disponibles) et qu'il convenait qu'elles soient le fruit d'une réflexion et d'une élaboration commune aux enseignants impliquées dans cette expérimentation. Il nous a paru indispensable de procéder d'abord à une sorte d'initiation à l'outil de référence, en l'occurrence la grille d'auto-évaluation du Cecr assortie de la liste de repérage croisant les 6 niveaux de compétence du Cadre (A1 à C2) avec les 5 compétences retenues (écouter, lire, prendre part à une conversation, s'exprimer oralement en continu, écrire). La première tâche consistait à expliciter et commenter les descripteurs de compétences (dits de type "can do") car la perspective actionnelle dans laquelle se placent les documents de référence du Conseil de l'Europe commence seulement à être diffusée dans de nombreux pays hors d'Europe. Après avoir formulé les descripteurs correspondant à leur public spécifique, les enseignants devaient se mettre d'accord sur la gradation retenue, en proposant des formulations et des degrés différents. L'apprenant peut ainsi être appelé à se positionner dans l'une des quatre colonnes proposées (de type "je suis capable de faire cela jamais/parfois/souvent/toujours"), voire parmi cinq colonnes (de "très difficilement" à "très facilement"). Des combinaisons binaires sont également possibles, comme dans le portfolio suisse : "je peux faire cela dans des circonstances normales/je peux faire cela bien et facilement" et "ceci est un objectif pour moi/ceci est prioritaire pour moi", etc. Pour les enfants, le choix peut être fait sur la base d'icônes significatifs (type "smiley" : :, , : , :)

Concernant la mise en application et pour éviter les critiques liées au manque d'objectivité des apprenants, nous avons souhaité coupler l'utilisation de l'outil retenu avec un processus de co-évaluation. Les enseignants devaient donc, en sus des classiques bulletins de note, remplir une fiche "miroir" dans laquelle ils se prononçaient sur la capacité de l'apprenant à accomplir les tâches décrites. Dans un premier temps, ce type de dispositif a semblé alourdir significativement la démarche. Cependant, au fil des mois, les enseignants ont pu mesurer 
les écarts possibles entre leur évaluation et celle des étudiants et constater que les différences n'étaient pas aussi marquées qu'ils le supposaient au départ. Cette démarche a donc contribué à donner une réelle légitimité à l'auto-évaluation dans les programmes de français de différentes institutions iraniennes, et ces efforts initiaux ont encouragé une véritable implication des différents acteurs. Sans cette implication, il est effectivement difficile d'envisager une évolution des opinions et attitudes liées à l'auto-évaluation, souvent teintées de scepticisme. Avec le recul, il ne fait pas de doute que si le Pepelf avait été disponible à l'époque, il aurait constitué un excellent document de support lors des sessions de formation continue des enseignants iraniens.

\section{Perspectives conclusives}

En guise de bilan, nous retiendrons que la valorisation de trajectoires plurilingues individualisées et non cloisonnées au domaine scolaire contribue à un changement de paradigme en matière d'enseignement-apprentissage qui ne va pas sans rencontrer freins et blocages, tout à la fois transversaux et spécifiques à chaque contexte éducatif particulier.

Si de nombreux Etats partagent aujourd'hui le postulat du Conseil de l'Europe selon lequel "développer sa compétence plurilingue, c'est apprendre à apprendre les langues et devenir capable de gérer son acquisition des langues de manière autonome et réflexive" (Beacco \& Byram, 2007 : 49), il faut donc envisager un processus de longue haleine et une inévitable implication des enseignants dans le même type de démarche. Tout enseignement de langue ne doit plus être envisagé comme une fin en soi, mais prendre en compte une sensibilisation aux stratégies d'apprentissage. La généralisation du Pepelf et du PEL en Europe va clairement dans ce sens en donnant la possibilité d'exploiter la multiplicité des formes possibles d'acquisition ou d'enseignement.

C'est la raison pour laquelle la diffusion et la mise en pratique généralisée de ce type d'outils s'inscrit parmi les priorités affichées par la Commission Européenne ces 15 dernières années en matière d'amélioration de la qualité des études et de la formation des enseignants. Le Livre Blanc "Enseigner et apprendre. Vers la société cognitive" adopté en 1995 pose comme objectif général la maîtrise de trois langues communautaires, soit la langue maternelle et deux langues étrangères. Il devient un slogan à forte valeur communicative et 
trouve des prolongements directs et concrets dans le plan d'action établi pour 2004-2006 visant à promouvoir l'apprentissage des langues et la diversité linguistique. Il complète ainsi les grands programmes déjà mis en place et couvrant un large spectre : Comenius pour les écoles, Erasmus pour les universités, Leonardo pour la formation professionnelle et Grundtvig pour l'enseignement aux adultes. En 2005, une direction spécifique est créée au sein de la Commission Européenne : elle est exclusivement consacrée au multilinguisme, et répond à des objectifs communs à ceux du Conseil de l'Europe. Le "Nouveau cadre stratégique pour le multilinguisme" (Commission of the European Communities, 2005) stipule que les programmes et les structures de la formation des enseignants de langues étrangères doivent tenir compte de l'évolution des besoins en matière de compétences que les élèves et les étudiants sont supposés acquérir. C'est dans ce cadre que la Commission a financé l'étude sur les "bonnes pratiques" en Europe qui donnera naissance au tronc commun de compétences et de valeurs propres aux enseignants européens à l'origine du Pepelf. Depuis, différentes communications et rapports (Commission of the European Communities, 2007a et b en particulier) ont été consacrés aux liens entre enseignement des langues et promotion de la diversité linguistique. Il est spécifiquement déclaré que la formation initiale n'est actuellement pas en mesure de donner aux enseignants toutes les connaissances requises durant leur carrière complète. En conséquence, "la formation et le perfectionnement professionnel de chaque enseignant doivent être considérés comme la tâche de toute une existence et bénéficier des structures et ressources adéquates" (Commission of the European Communities, 2007a : 13). Cet objectif est d'autant plus important que l'évolution de la société impose de nouvelles exigences à l'enseignant et lui confère donc un nouveau rôle : il n'est plus seulement censé dispenser des connaissances élémentaires, mais il lui est de plus en plus demandé d'aider les jeunes à devenir des apprenants autonomes grâce à l'acquisition de compétences clés, plutôt que par la mémorisation d'informations. En application des théories socio-constructivistes, l'enseignant doit favoriser des méthodes d'acquisition davantage axées sur la collaboration entre pairs et la co-construction des savoirs. Le rapport qualitatif attendu à la fin du projet pilote du Pepelf devrait donc permettre de valoriser ce nouvel outil de construction d'une éducation plurilingue tout au long de la vie : il plaide en effet pour une formation spécifique des enseignants de langue de l'enseignement supérieur assortie d'une période obligatoire de formation dans un autre pays 
permettant de développer des compétences interculturelles. Cet instrument de référence, à l'instar du PEL, devrait logiquement constituer dans les années à venir et dans des versions adaptées, un élément-clé des politiques éducatives en Europe et sur d'autres continents. Pour autant, il ne faudrait pas se laisser aller à la tentation de la simple incantation: le plurilinguisme n'ira pas de soi si l'on se contente de faire seulement référence à ces portfolios ou de procéder à leur application systématique. Seuls une réflexion de long terme et un accompagnement des acteurs impliqués pourront permettre aux apprenants de prendre confiance dans leurs propres capacités à développer leur connaissance des langues et à tirer parti des stratégies d'apprentissage en vue de s'approprier des variétés de langues non connues à partir de variétés connues et d'accroître ainsi leur motivation à apprendre tout au long de la vie. 


\section{Références}

\section{Bibliographie}

Abric, J.-C. (1994). "L'organisation interne des représentations sociales : système central et système périphérique". In Guimelli, C. Structures et transformations des représentations sociales. Neuchatel : Delachaux et Niestlé.

Allal, L. (1988). "Vers un élargissement de la pédagogie de la maîtrise : processus de régulation interactive, rétroactive et proactive". In Huberman, M. (éd.). Assurer la réussite des apprentissages scolaires? Les propositions de la pédagogie de la maîtrise. Neuchatel : Delachaux et Niestlé.

Allal, L. (2011). "Pedagogy, didactics and the co-regulation of learning: a perspective from the French-language world of educational research", Research Papers in Education, 26:3. pp. 329-336.

Barbot, M.-J. (1990). "Métamorphoses de l'évaluation: l'évaluation dans les systèmes d'auto-apprentissage", Etudes de Linguistique Appliquée, nº 80, p. 77-99.

Beacco, J.-C. (2005). Langues et répertoires de langues : le plurilinguisme comme "manière d'être" en Europe. Strasbourg : Conseil de l'Europe.

Beacco, J.-C. \& Byram, M. (2007). De la diversité linguistique à l'éducation plurilingue: guide pour l'élaboration des politiques linguistiques éducatives en Europe. Strasbourg: Conseil de l'Europe.

Blanchard-Laville, C. \& Fablet, D. (coord.). (2001). Sources théoriques et techniques de l'analyse de pratiques professionnelles. Paris : L'Harmattan.

Bourdieu, P. (1972). Esquisse d'une théorie de la pratique. Genève : Droz.

Cambra Giné, M. (2003). Une approche ethnographique de la classe de langue. Paris : Didier. 
Campanale, F. (1997). "Auto-évaluation et transformation de pratiques pédagogiques". Mesure et évaluation en éducation, vol. 20, n¹. pp. 1-24.

Castellotti, V. \& Moore, D. (2006). "Parcours d'expériences plurilingues et conscience réflexive". Le français dans le monde, Recherches et Applications, n $^{\circ}$ 39, pp. 54-68.

Castellotti, V. \& Moore, D. (2002). Représentations sociales des langues et enseignement. Strasbourg : Division des Politiques Linguistiques, Conseil de l'Europe.

Commission Européenne. (1995). Enseigner et apprendre, vers la société cognitive, Livre blanc sur l'éducation et la formation. Luxembourg : OPOCE.

Commission of the European Communities. (2005). COM(2005)596: A New Framework Strategy for Multilingualism. Disponible en ligne: http://ec.europa.eu/education/policies/lang/doc/com596_en.pdf

Commission of the European Communities. (2007a). COM(2007)392: Improving the Quality of Teacher Education. Disponible en ligne: http://ec.europa.eu/education/com392_en.pdf

Commission of the European Communities. (2007b). COM(2007)554: rapport sur la mise en œuvre du plan d'action "Promouvoir l'apprentissage des langues et la diversité linguistique". Disponible en ligne : http://eur-lex.europa.eu/LexUriServ/LexUriServ.do?uri=COM:2007:0554:FIN:FR:PDF

Coste, D., Moore, D. \& Zarate, G. (1997). Compétence plurilingue et pluriculturelle. Strasbourg : Conseil de l'Europe.

Division des Politiques Linguistiques. (2000). Portfolio Européen des Langues, principes et lignes directrices. Strasbourg : Conseil de l'Europe.

Duda, R. \& Riley, P. (1990). Learning Styles. Nancy : Presses Universitaires de Nancy.

Flament, C. (1999). "Structure et dynamique des représentations sociales". In Jodelet, D. (dir.). Les représentations sociales. Paris : PUF. 
Grandcolas, B. \& Vasseur, M.-T. (1999). "Conscience d'enseignant, conscience d'apprenant, réflexions interactives pour la formation". Socrates/Lingua Action A n 25043-CP-2-97-FRLINGUA-LA.

Harris, M. (1997). "Self-assessment of language learning in formal settings ", ELT Journal, Volume 51/1.pp. 12-20.

Holec, H. (1991). "Autonomie de l'apprenant: de l'enseignement à l'apprentissage". Education permanente, $\mathrm{n}^{\circ} 107 . \mathrm{pp} .1-5$.

Holec, H. (1981). Autonomie et apprentissage des langues étrangères. Paris : Hatier.

Little, D. (2009). "Language learner autonomy and the European Language Portfolio: Two L2 English examples", Language Teaching, 42:2. pp. 222-233.

Moscovici, S. (1961). La psychanalyse, son image et son public. Paris: PUF.

Newby, D. (2007). "The European Portfolio for Student Teachers of Languages". Babylonia, vol. 3/2007, pp. 1-6.

Normand-Marconnet N. (2008). L'auto-évaluation : évidence, défi ou utopie? Vers une nouvelle relation enseignant-enseigné mettant en jeu les variétés de contexte et de culture éducative : le cas de l'Iran. Thèse de doctorat soutenue en 2008 à l'Université du Maine, non publiée.

Nunan, D. (1999). Second language teaching and learning. Boston : Heine and Heine Publishing.

Perrenoud, P. (2001). "De la pratique réflexive au travail sur l'habitus". Recherche et formation, $\mathrm{n}^{\circ} 36$, pp.131-162.

Schärer, R. (2000). Rapport final - Portfolio Européen des Langues, phase pilote 19982000. Conseil de l'Europe, CDCC, Comité de l'Education, DGIV/EDU/LANG(2000)31-rev. Disponible en ligne : http://www.coe.int/t/dg4/education/elp/elpreg/Source/History/ELP_PilotProject_FinalReport_FR.pdf

Scriven, M. (1967). "The Methodology of Evaluation", AERA Monograph Series on Evaluation, ${ }^{\circ} 1$.pp. 39-83. 
Les Cahiers de l'Acedle, volume 9, numéro 1, 2012

Recherches en didactique des langues et cultures

Wenden, A. (2002). "Learner Development in Language Learning". Applied Linguistics, vol. 23, n 1 . pp. 32-55. 


\section{Annexes}

Tableau 1-Questions de type échelle

\begin{tabular}{|c|c|}
\hline Je pense qu'il est nécessaire de continuer & $\begin{array}{l}\text { Moyenne } \\
\text { cumulée }\end{array}$ \\
\hline pour faire réfléchir les apprenants à leur apprentissage & 1,31 \\
\hline pour améliorer l'ensemble des programmes & 1,41 \\
\hline parce que cela m'amène à réfléchir à mes pratiques d'enseignement & 1,42 \\
\hline pour améliorer mes propres cours & 1,53 \\
\hline pour motiver les apprenants & 1,76 \\
\hline pour associer les apprenants à la démarche d'évaluation & 1,79 \\
\hline pour établir une relation différente avec mes apprenants & 1,84 \\
\hline parce que c'est une nouveauté pédagogique qu'il faut connaître & 1,84 \\
\hline pour impliquer plus les enseignants dans le fonctionnement de l'institut & 1,86 \\
\hline $\begin{array}{l}\text { parce que cela peut inciter d'autres instituts ou organismes d'enseignement à mettre en place cette } \\
\text { démarche d'auto-évaluation qui n'est pas très connue en Iran }\end{array}$ & 2,43 \\
\hline \multicolumn{2}{|l|}{ Je pense que cette démarche d'auto-évaluation n'est pas adaptée à l'Iran } \\
\hline parce que les apprenants donnent plus d'importance aux examens et aux notes & 1,92 \\
\hline parce que les apprenants ne sont pas habitués à réfléchir à ces questions et à donner leur avis & 2,35 \\
\hline parce que les apprenants ne sont pas objectifs sur leur niveau & 2,81 \\
\hline parce que les apprenants ne comprennent pas à quoi ça sert & 2,97 \\
\hline parce que seul l'enseignant est expérimenté pour juger le niveau des apprenants & 3,15 \\
\hline \multicolumn{2}{|l|}{ Je pense que pour que cette démarche se développe en Iran, il faudrait } \\
\hline $\begin{array}{l}\text { que les enseignants qui commencent à se familiariser avec l'auto-évaluation introduisent peu à peu } \\
\text { cette démarche dans les instituts, écoles et universités où ils travaillent, sans attendre l'appui du } \\
\text { ministère }\end{array}$ & 1,52 \\
\hline $\begin{array}{l}\text { que les enseignants du public et du privé soient sensibilisés et formés par le ministère et des } \\
\text { experts étrangers }\end{array}$ & 1,90 \\
\hline qu'elle soit imposée dans les programmes scolaires par le ministère de l'Education & 1,98 \\
\hline
\end{tabular}


Tableau 2 - Questions fermées

\begin{tabular}{|l|c|c|c|}
\hline & $\%$ oui & \% non Je ne sais pas \\
\hline J'ai envie de continuer cette démarche de façon autonome & 53 & 18 & 29 \\
\hline $\begin{array}{l}\text { J'ai envie de continuer seulement si la direction des cours continue à } \\
\text { piloter le projet }\end{array}$ & 45 & 33 & 22 \\
\hline Je n'ai pas envie de continuer & 10 & 65 & 25 \\
\hline J'ai l'impression d'avoir une relation différente avec mes apprenants & 51 & 16 & 33 \\
\hline J'ai l'impression que mes apprenants me considèrent autrement & 49 & 14 & 38 \\
\hline J'aimerais avoir des séances de discussion sur l'auto-évaluation & 88 & 2 & 10 \\
\hline J'aimerais suivre des ateliers de formation sur l'auto-évaluation & 86 & 0 & 14 \\
\hline
\end{tabular}

\section{À propos de l'auteure}

Nadine Normand-Marconnet est actuellement maîtresse de conférences à l'université Monash (Melbourne, Australie). Elle a soutenu sa thèse en Sciences du langage à l'université du Maine en 2008 ; parallèlement, elle a poursuivi ses activités de conseiller technique en éducation pour le Ministère des Affaires étrangères et européennes jusqu'à son recrutement en Australie en janvier 2011 sur un poste d'enseignant-chercheur au sein du département d'Études Françaises.

Courriel : nadine.normandmarconnet@monash.edu

Toile : http://arts.monash.edu/french/staff/nmarcon.php

Adresse : Monash University, School of Languages, Cultures and Linguistics, VIC 3800, Australia. 